\title{
Critical Values for Testing Location-Scale Hypothesis
}

\author{
František Rublík \\ Institute of Measurement Science, Slovak Academy of Sciences, Dúbravská cesta 9, 84104 Bratislava, Slovakia \\ E-mail: umerrubl@ savba.sk
}

The exact critical points for selected sample sizes and significance levels are tabulated for the two-sample test statistic which is a combination of the Wilcoxon and the Mood test statistic. This statistic serves for testing the null hypothesis that two sampled populations have the same location and scale parameters.

Keywords: distribution-free rank test; combination of rank statistics; small sample sizes; location-scale hypothesis.

\section{INTRODUCTION}

$\mathbf{S}$

UPPOSE THAT the random variables $X, Y$ have the distribution functions

$$
P(X \leq t)=F\left(\frac{t-\mu_{X}}{\sigma_{X}}\right), \quad P(Y \leq t)=F\left(\frac{t-\mu_{Y}}{\sigma_{Y}}\right),
$$

where the real numbers $\mu_{X}, \mu_{Y}$ denote the location parameters, $\sigma_{X}>0, \sigma_{Y}>0$ are scale parameters and $F$ is a continuous distribution function defined on the real line. Assume that $X_{1}, \ldots, X_{m}$ is a random sample from the distribution of $X, Y_{1}, \ldots, Y_{n}$ is a random sample from the distribution of $Y$ and these random samples are independent. Let $\left(R_{1}, \ldots, R_{N}\right), N=m+n$, denote the ranks of the pooled sample $\left(X_{1}, \ldots, X_{m}, Y_{1}, \ldots, Y_{n}\right)$. The hypothesis

$$
H_{0}: \mu_{X}=\mu_{y}, \quad \sigma_{X}=\sigma_{Y}
$$

is sometimes called also the null location-scale hypothesis. If the populations $X$ and $Y$ differ in their location parameters only, then the tests designed for detecting this change (e.g. Wilcoxon-Mann-Whitney or van der Waerden test) can be used to detect this difference. Similarly, if $X$ and $Y$ differ in the scale parameter only, then the tests designed for detecting this change (e.g. Mood or Ansari-Bradley test) can be used. However, the tests designed for detecting difference of the location usually do not perform well when the change occurs in the scale parameter only. Similarly, the tests designed for detecting difference of the scale usually do not perform well when the change occurs in the location parameter only. But in the real situation it is not known which type of change will occur and if the experimenter is interested both in the difference of the location and also in the difference of scale, the use of the test statistics mentioned before will not be advantageous. This is due to the fact that such a difference cannot be simply detected in the one step, and the two step use of the mentioned tests would lead to the increase of the nominal level of significance (the probability of the error of type I). But what is even more important is the fact, that in practice the change in the location is very often accompanied by the change in scale, and in such a case the statistics constructed especially for testing the location-scale null hypothesis (2) usually yield better results than the statistics constructed especially for the one type change of location or constructed especially for the one type change of scale. Readers interested in further discussion of the need of testing the hypothesis (2) can found further arguments in Section 1 of [6] or in [3].

We shall deal with the test based on the ranks of the observations. As is well known, the advantage of such a test is that in the setting (1) with $F$ continuous, the use of this rank test guarantees that the significance level will have the chosen value $\alpha$. The null hypothesis (2) is against the alternative $H_{1}$ that at least one of the equalities (2) does not hold, tested usually by means of the Lepage test from [4]. Critical constants of this test for small sample sizes can be found in [5], the Lepage test is included also into the monograph [2]. The Lepage test statistic is given by the formula

$$
\begin{gathered}
T=T_{K}+T_{B}, \\
T_{K}=\frac{\left(S_{W}-E\left(S_{W} \mid H_{0}\right)\right)^{2}}{\operatorname{Var}\left(S_{W} \mid H_{0}\right)}, \quad T_{B}=\frac{\left(S_{B}-E\left(S_{B} \mid H_{0}\right)\right)^{2}}{\operatorname{Var}\left(S_{B} \mid H_{0}\right)},
\end{gathered}
$$

where $S_{W}=\sum_{i=1}^{m} R_{i}$ is the Wilcoxon rank test statistic and $S_{B}=\sum_{i=}^{m} a_{N}\left(R_{i}\right)$ is the Ansari-Bradley rank test statistic (i.e., the vector of scores $a_{N}=(1,2, \ldots, k, k, \ldots, 2,1)$ if $N=2 k$ and $a_{N}=(1,2, \ldots, k, k+1, k, \ldots, 2,1)$ if $\left.N=2 k+1\right)$. An analogous statistic has been formulated in the multisample setting in [7], another test statistics for this problem have been studied in [8], where also their non-centrality parameters for testing the null location-scale hypothesis are for some situations computed. As shown on p. 283 of [8], the bounds for the asymptotic efficiency in the case of there considered sampled distributions and quadratic rank statistics do not depend on the number of sampled populations. As concluded ibidem, taking into account computed values of the asymptotic efficiencies, one sees that a combination of the multisample Kruskal-Wallis statistic (in the two-sample case the Wilcoxon test statistic) with the Mood test statistic appears to be a good choice when one considers symmetric distributions whose type of tail weight is unknown. The statistics in [8] are defined in the general multisample setting, but they can be used also in the two-sample setting for small sample sizes, when the tables of the critical constants can be computed. However, the only available tables of critical constants 
for testing the location scale-hypothesis are those published in [5]. The mentioned table is computed for $m=2(1) 30, n=2$; $\mathrm{m}=3(1) 26, \mathrm{n}=3 ; \mathrm{m}=4(1) 18, \mathrm{n}=4 ; \mathrm{m}=5(1) 13, \mathrm{n}=5 ; \mathrm{m}=6(1) 10$, $\mathrm{n}=6 ; \mathrm{m}=7,8, \mathrm{n}=7$ and the significance levels $\alpha=0.01,0.02$, $0.05,0.10$ and 0.2 . In this paper also the significance level $\alpha=0.005$ is included, the extremely unbalanced sample sizes like $m=2, n=30$ are not considered and instead of these critical constants for some other small sample sizes for $m=7(1) 10$ are computed.

\section{BASIC FORMULAS}

The topic of this paper is the computation of the critical values for the two-sample test statistic (4). In accordance with [8] label the combination of the Wilcoxon and the Mood statistic by $T_{S Q}$. Thus in the notation from the previous section

$$
\begin{aligned}
T_{S Q} & =T_{K}+Q, \\
T_{K} & =\frac{12}{m n(N+1)}\left(S_{W}-\frac{m(N+1)}{2}\right)^{2}, \\
Q & =\frac{180}{m n(N+1)\left(N^{2}-4\right)}\left(\tilde{S}-\frac{m\left(N^{2}-1\right)}{12}\right)^{2}, \\
\tilde{S} & =\sum_{i=1}^{m}\left(R_{i}-\frac{N+1}{2}\right)^{2} .
\end{aligned}
$$

Since according to the assumptions the distribution function $F$ in (1) is continuous, the statistic $T_{S Q}$ is distribution-free whenever the null hypothesis (2) holds. The null hypothesis (2) is rejected whenever $T_{S Q} \geq w_{\alpha}$. Values of $w_{\alpha}=w_{\alpha}(m, n)$ can be found in the table presented in the next section, for the sample sizes not included into the table instead of $w_{\alpha}$ use the $(1-\alpha)$ th quantile of the chi-square distribution with 2 degrees of freedom.

In the computation of the tables of this paper the following lemma is useful.

Lemma 1. Let $J(m, n)$ denote the set of all $m$-tuples $\left(i_{1}, \ldots, i_{m}\right)$ consisting of integers such that $1 \leq i_{1}<\ldots<$ $i_{m} \leq m+n$. Suppose that

$$
\begin{aligned}
D\left(m, n, k_{1}, k_{2}\right) & =\left\{\left(i_{1}, \ldots, i_{m}\right)\right. \\
& \left.\in J(m, n) ; \sum_{j=1}^{m} i_{j}=k_{1}, \sum_{j=1}^{m} i_{j}^{2}=k_{2}\right\},
\end{aligned}
$$

and $B\left(m, n, k_{1}, k_{2}\right)$ denotes the number of elements of $D\left(m, n, k_{1}, k_{2}\right)$.

(I) Let $s>1$. If at least one of the inequalities $k_{1} \leq(r+s)$, $k_{2} \leq(r+s)^{2}$ holds, then $B\left(s, r, k_{1}, k_{2}\right)=B\left(s, r-1, k_{1}, k_{2}\right)$.

(II) If $k_{1}>(s+r), k_{2}>(s+r)^{2}$, then $B\left(s, r, k_{1}, k_{2}\right)=$ $B\left(s, r-1, k_{1}, k_{2}\right)+B\left(s-1, r, k_{1}-(r+s), k_{2}-(r+s)^{2}\right)$.

Proof. (I) If $k_{1} \leq r+s$, then $\sum_{j=1}^{s} i_{j}=k_{1}$ if and only if this equality holds and $(r+s) \notin\left\{i_{1}, \ldots, i_{s}\right\}$. Similarly, if $k_{2} \leq(r+s)^{2}$, then $\sum_{j=1}^{s} i_{j}^{2}=k_{2}$ if and only if this equality holds and $(r+s) \notin\left\{i_{1}, \ldots, i_{s}\right\}$.

(II) This assertion follows from the fact, that $D\left(s, r, k_{1}, k_{2}\right)$ is a union of the disjoint sets $D\left(s, r-1, k_{1}, k_{2}\right)$ and $\left\{\left(i_{1}, \ldots, i_{s}\right) \in J(r, s) ; i_{s}=s+r, \sum_{j=1}^{s} i_{j}=k_{1}, \sum_{j=1}^{s} i_{j}^{2}=k_{2}\right\}$.

If $R=\left(R_{1}, \ldots, R_{N}\right)$ is a random vector which is uniformly distributed over the set of all permutations of the set $\{1, \ldots, N\}$, then according to Theorem 1 on p. 167 of [1] for any set $A \subset\{1, \ldots, N\}$ consisting of $m$ distinct integers

$$
P\left(\left\{R_{1}, \ldots, R_{m}\right\}=A\right)=1 /\left(\begin{array}{l}
N \\
m
\end{array}\right) .
$$

Combining this equality with Lemma 1 one can construct a program for computation of critical values of the statistic (4).

\section{TABLES OF CRITICAL VALUES}

In this section we present the exact critical values of the statistic $T_{S Q}$ from (4), given in Table 2. First we describe the output of the table.

Since the set $V=V(m, n)$ of possible values of the statistic $T_{S Q}$ is finite for all sample sizes $m, n$, generally one cannot find exact critical values for arbitrary prescribed probability $\alpha$ of the type $I$ error. In the following table the number on the intersection of the column for $\underline{w}$ with the row for significance level $\alpha$ denotes the quantity

$$
\underline{w}=\min \left\{t \in V ; P\left(T_{S Q} \geq t\right) \leq \alpha\right\},
$$

the entry corresponding to $\alpha$ and $\bar{w}$ is the quantity

$$
\bar{w}=\max \left\{t \in V ; P\left(T_{S Q} \geq t\right)>\alpha\right\} .
$$

Further, for given $\alpha$,

$$
\underline{p}=P\left(T_{S Q} \geq \underline{w}\right), \quad \bar{p}=P\left(T_{S Q} \geq \bar{w}\right)
$$

denote the corresponding probabilities of the type I error. Thus $p$ is the largest available significance level not exceeding $\alpha$ and $\bar{p}$ is the smallest available significance level greater than $\alpha$. The value of critical constant yielding the significance level closer to the nominal level $\alpha$ is printed in boldface letter. If the difference in computed values exceeds the number of decimal places used to describe the result of computation, then the boldface symbol is used for the value corresponding to $p$.

\section{Some Simulation RESUlts}

The aim of the simulation is to obtain a picture of the power of tests based on the statistics (3) and (4) for small sample sizes covered by Table 2 . To consider power of the concerned tests in the case of distributions with various tail behaviour, the sampling from normal, logistic and Cauchy distribution is employed, in each case $\mu_{X}=0, \sigma_{X}=1$. The simulations are 
Table 1: Simulation estimates of the power.

\begin{tabular}{|c|c|c|c|c|c|c|}
\hline & \multicolumn{6}{|c|}{$m=3, n=7$} \\
\hline & \multicolumn{2}{|c|}{$\mu_{Y}=1.5, \sigma_{Y}=3$} & \multicolumn{2}{|c|}{$\mu_{Y}=3.5, \sigma_{Y}=4$} & \multicolumn{2}{|c|}{$\mu_{Y}=7, \sigma_{Y}=5$} \\
\hline & $\alpha=0.05$ & $\alpha=0.10$ & $\alpha=0.05$ & $\alpha=0.10$ & $\alpha=0.05$ & $\alpha=0.10$ \\
\hline$P\left(T_{S Q} \geq w_{\alpha} \mid\right.$ Normal $)$ & 0.057 & 0.073 & 0.202 & 0.224 & 0.533 & 0.547 \\
\hline$P(T \geq$ t $\alpha \mid$ Normal $)$ & 0.032 & 0.141 & 0.136 & 0.251 & 0.435 & 0.539 \\
\hline$P\left(T_{S Q} \geq w_{\alpha} \mid\right.$ Logistic $)$ & 0.032 & 0.041 & 0.073 & 0.086 & 0.191 & 0.208 \\
\hline$P(T \geq t \alpha \mid$ Logistic $)$ & 0.017 & 0.131 & 0.047 & 0.170 & 0.137 & 0.247 \\
\hline$P\left(T_{S Q} \geq w_{\alpha} \mid\right.$ Cauchy $)$ & 0.041 & 0.058 & 0.084 & 0.104 & 0.174 & 0.197 \\
\hline \multirow[t]{4}{*}{$P(T \geq t \alpha \mid$ Cauchy $)$} & 0.025 & 0.114 & 0.060 & 0.165 & 0.138 & 0.233 \\
\hline & \multicolumn{6}{|c|}{$m=7, n=8$} \\
\hline & \multicolumn{2}{|c|}{$\mu_{Y}=1.5, \sigma_{Y}=3$} & \multicolumn{2}{|c|}{$\mu_{Y}=3.5, \sigma_{Y}=4$} & \multicolumn{2}{|c|}{$\mu_{2}=7, \sigma_{2}=5$} \\
\hline & $\alpha=0.05$ & $\alpha=0.10$ & $\alpha=0.05$ & $\alpha=0.10$ & $\alpha=0.05$ & $\alpha=0.10$ \\
\hline$P\left(T_{S Q} \geq w_{\alpha} \mid\right.$ Normal $)$ & 0.448 & 0.640 & 0.745 & 0.886 & 0.950 & 0.987 \\
\hline$P(T \geq t \alpha \mid$ Normal $)$ & 0.415 & 0.605 & 0.730 & 0.868 & 0.952 & 0.984 \\
\hline$P\left(T_{S Q} \geq w_{\alpha} \mid\right.$ Logistic $)$ & 0.363 & 0.540 & 0.586 & 0.765 & 0.796 & 0.915 \\
\hline$P(T \geq t \alpha \mid$ Logistic $)$ & 0.326 & 0.510 & 0.547 & 0.732 & 0.774 & 0.900 \\
\hline$P\left(T_{S Q} \geq w_{\alpha} \mid\right.$ Cauchy $)$ & 0.228 & 0.323 & 0.431 & 0.566 & 0.659 & 0.759 \\
\hline$P(T \geq$ t $\alpha \mid$ Cauchy $)$ & 0.219 & 0.356 & 0.419 & 0.568 & 0.643 & 0.757 \\
\hline
\end{tabular}

based in each case on 10000 trials. The critical constants $w_{\alpha}$ of the statistic $T_{S Q}$ defined in (4) are taken from Table 2, the critical constants $t_{\alpha}$ of the statistic (3) are those computed in [5]. The results of simulations are in Table 1 . The power better of the two considered cases is emphasized by the boldface type.

\section{Discussion AND CONCLUSIONS}

As shown on p. 283 of [8], the bounds for the asymptotic efficiency of the test statistics (3) and (4) in the case of there considered sampled distributions do not depend on the number of sampled populations, i.e., they are the same in the two-sample case and in the multisample case. As concluded in [8], taking into account computed values of the asymptotic efficiencies, one sees that a combination of the multisample Kruskal-Wallis statistic (in the two-sample case the Wilcoxon test statistic) with the Mood test statistic appears to be a good choice when one considers symmetric distributions whose type of tail weight is unknown. However, these considerations are related to the asymptotic case, when both $m$ and $n$ tend to infinity. The simulation results, given in Table 1 do not contradict the mentioned asymptotic results. After inspecting Table 1 it can be said that for small sample sizes and $\alpha=0.05$ testing based on (4) is preferable to (3), but for $\alpha=0.1$ it is advisable to use the Lepage test. This suggests that the test based on (4) can be considerered as a useful competitor to the
Lepage test.

\section{ACKNOWLEDGMENTS}

The research was supported by the grants VEGA 1/0077/09, APVV-SK-AT-0003-09, and APVV-RPEU-0008-06.

\section{REFERENCES}

[1] Hájek, J., Šidák, Z. \& Sen, P. K. (1999). Theory of Rank Tests. Academic Press, San Diego.

[2] Hollander, M. \& Wolfe, D. A. (1999). Nonparametric Statistical Methods. Wiley, New York.

[3] Hutchison, T. P. (2002). Should we routinely test for simultaneous location and scale changes? Ergonomics 45, 248-251.

[4] Lepage, Y. (1971). A combination of Wilcoxon's and AnsariBradley's statistics. Biometrika 58, 213-217.

[5] Lepage, Y. (1973). A table for a combined Wilcoxon AnsariBradley statistic. Biometrika 60, 113-116.

[6] Podgor, M. J. \& Gastwirth, J. L. (1994). On non-parametric and generalized tests for the two-sample problem with location and scale change alternatives. Statistics in Medicine 13, 747-758.

[7] Rublík, F. (2005). The multisample version of the Lepage test. Kybernetika 41, 713-733.

[8] Rublík, F. (2007). On the asymptotic efficiency of the multisample location-scale rank tests and their adjustment for ties. Kybernetika 43, 279-306. 
Table 2: Critical values of the test statistic $T_{S Q}$ from (4).

\begin{tabular}{|c|c|c|c|c|c|c|c|c|c|c|c|c|}
\hline$\alpha$ & $\underline{w}$ & $\underline{p}$ & $\bar{w}$ & $\bar{p}$ & $\underline{w}$ & $\underline{p}$ & $\bar{w}$ & $\bar{p}$ & $\underline{w}$ & $\underline{p}$ & $\bar{w}$ & $\bar{p}$ \\
\hline & & $\mathrm{m}=3$ & $n=3$ & & & $m=3$ & $\mathrm{n}=4$ & & & $m=3$ & $\mathrm{n}=5$ & \\
\hline 0.200 & 3.8571 & .100 & 3.6428 & .300 & 4.1666 & .143 & 3.1666 & .257 & 3.9555 & .143 & 2.8888 & .214 \\
\hline 0.100 & 3.8571 & .100 & 3.6428 & .300 & & & 4.6666 & .114 & 5.5555 & .071 & 3.9555 & .143 \\
\hline 0.050 & & & 3.8571 & .100 & & & 4.6666 & .114 & & & 5.5555 & .071 \\
\hline 0.010 & & & 3.8571 & .100 & & & 4.6666 & .114 & & & 5.5555 & .071 \\
\hline \multirow[t]{2}{*}{0.005} & & & 3.8571 & .100 & & & 4.6666 & .114 & & & 5.5555 & .071 \\
\hline & & $\mathrm{m}=3$ & $\mathrm{n}=6$ & & & $\mathrm{~m}=3$ & $\mathrm{n}=7$ & & & $\mathrm{~m}=3$ & $\mathrm{n}=8$ & \\
\hline 0.200 & 3.5520 & .179 & 3.2796 & .202 & 3.1298 & .2 & 3.0519 & .217 & 3.3803 & .176 & 3.2435 & .212 \\
\hline 0.100 & 4.2077 & .083 & 3.6121 & .107 & 4.2532 & .100 & 4.0454 & .117 & 4.1880 & .091 & 4.0213 & .103 \\
\hline 0.010 & & & 6.4519 & .024 & & & 7.3181 & .017 & & & 8.1367 & .012 \\
\hline \multirow[t]{2}{*}{0.005} & & & 6.4519 & .024 & & & 7.3181 & .017 & & & 8.1367 & .012 \\
\hline & & $\mathrm{m}=3$ & $\mathrm{n}=9$ & & & $m=3$ & $\mathrm{n}=10$ & & & $m=3$ & $\mathrm{n}=11$ & \\
\hline 0.200 & 3.2649 & .191 & 3.1184 & .200 & 3.0649 & .199 & 3.0026 & .203 & 3.0363 & .198 & 2.9454 & .203 \\
\hline 0.100 & 4.1147 & .082 & 4.0659 & .100 & 4.1558 & .094 & 3.8961 & .108 & 4.0621 & .099 & 4.0606 & .104 \\
\hline 0.050 & 5.5213 & .045 & 5.4434 & .055 & 5.7662 & .049 & 5.1532 & .056 & 5.1757 & .049 & 5.0303 & .055 \\
\hline 0.020 & 8.1098 & .018 & 6.9572 & .027 & 8.5714 & .014 & 7.6363 & .021 & 8.2787 & .016 & 6.8015 & .022 \\
\hline 0.010 & 8.9011 & .009 & 8.1098 & .018 & 9.6103 & .007 & 8.5714 & .014 & 10.2667 & .006 & 8.9833 & .011 \\
\hline \multirow[t]{2}{*}{0.005} & & & 8.9011 & .009 & & & 9.6103 & .007 & & & 10.2667 & .0055 \\
\hline & & $\mathrm{m}=3$ & $\mathrm{n}=12$ & & & $\mathrm{~m}=3$ & $\mathrm{n}=13$ & & & $m=3$ & $\mathrm{n}=14$ & \\
\hline 0.200 & 3.0754 & .185 & 3.0509 & .207 & 3.0101 & .196 & 2.9532 & .204 & 2.9507 & .200 & 2.9239 & .206 \\
\hline 0.020 & 7.3751 & .018 & 6.9951 & .022 & 7.5350 & .018 & 7.4057 & .021 & 7.7827 & .018 & 7.6424 & .021 \\
\hline 0.010 & 9.3529 & .009 & 8.8842 & .013 & 9.6862 & .007 & 9.4535 & .011 & 9.9883 & .009 & 8.4444 & .012 \\
\hline \multirow[t]{2}{*}{0.005} & 10.8733 & .0044 & 9.3529 & .0088 & 11.4342 & .0036 & 9.6862 & .0071 & 11.9532 & .0029 & 9.9883 & .0059 \\
\hline & & $m=3$ & $\mathrm{n}=15$ & & & $\mathrm{~m}=3$ & $\mathrm{n}=16$ & & & $m=3$ & $\mathrm{n}=17$ & \\
\hline 0.200 & 2.9745 & .196 & 2.9535 & .201 & 2.9600 & .198 & 2.9512 & .200 & 2.94899 & .198 & 2.94899 & .200 \\
\hline 0.100 & 4.1324 & .098 & 4.0342 & .103 & 4.0403 & .098 & 3.9953 & .100 & 3.9981 & .098 & 3.9879 & .100 \\
\hline 0.050 & 5.5500 & .049 & 5.3508 & .051 & 5.3113 & .050 & 5.2542 & .052 & 5.5137 & .049 & 5.4526 & .051 \\
\hline 0.020 & 7.7052 & .020 & 7.2552 & .022 & 7.7315 & .019 & 7.3407 & .021 & 7.5665 & .019 & 7.2454 & .021 \\
\hline 0.010 & 8.9403 & .010 & 8.5929 & .012 & 9.4113 & .008 & 9.0836 & .010 & 9.5494 & .009 & 8.7461 & .011 \\
\hline \multirow[t]{2}{*}{0.005} & 10.4904 & .0049 & 10.2632 & .0074 & 10.9618 & .0041 & 10.5143 & .0062 & 11.4046 & .0035 & 10.7446 & .0053 \\
\hline & & $m=3$ & $\mathrm{n}=18$ & & & $m=3$ & $\mathrm{n}=19$ & & & $m=3$ & $\mathrm{n}=20$ & \\
\hline 0.200 & 2.9361 & .200 & 2.9346 & .201 & 2.9725 & .197 & 2.9416 & .200 & 2.9438 & .199 & 2.9419 & .202 \\
\hline 0.100 & 3.9796 & .100 & 3.9680 & .102 & 4.0114 & .099 & 4.0034 & .100 & 4.0038 & .100 & 3.9342 & .101 \\
\hline 0.050 & 5.3942 & .050 & 5.3563 & .054 & $\mathbf{5 . 5 5 3 7}$ & .049 & 5.5308 & .051 & 5.4752 & .050 & 5.4142 & .051 \\
\hline 0.020 & 7.5072 & .020 & 7.2937 & .021 & 7.5125 & .019 & 7.4759 & .022 & 7.7419 & .019 & 7.7142 & .020 \\
\hline 0.010 & 9.0206 & .009 & 9.0135 & .011 & 9.2757 & .009 & 9.1235 & .010 & 9.4466 & .009 & 9.3609 & .010 \\
\hline 0.005 & 10.9565 & .0045 & 10.2827 & .0060 & 11.1522 & .0039 & 10.6854 & .0052 & 11.0676 & .0045 & 10.8086 & .0056 \\
\hline
\end{tabular}


Table 2 (cont.)

\begin{tabular}{|c|c|c|c|c|c|c|c|c|c|c|c|c|}
\hline$\alpha$ & $\underline{w}$ & $\underline{p}$ & $\bar{w}$ & $\bar{p}$ & $\underline{w}$ & $\underline{p}$ & $\bar{w}$ & $\bar{p}$ & $\underline{w}$ & $\underline{p}$ & $\bar{w}$ & $\bar{p}$ \\
\hline & & $\mathrm{m}=3$ & $\mathrm{n}=21$ & & & $\mathrm{~m}=3$ & $\mathrm{n}=22$ & & & $\mathrm{~m}=3$ & $n=23$ & \\
\hline 0.200 & 2.9728 & .199 & 2.9421 & .201 & 2.9334 & .200 & 2.9296 & .201 & 2.9329 & .200 & 2.9314 & .201 \\
\hline 0.100 & 4.0028 & .100 & 3.9893 & .101 & 4.0059 & .100 & 3.9927 & .100 & 4.0080 & .099 & 3.9974 & .100 \\
\hline 0.050 & 5.4801 & .049 & 5.4174 & .050 & 5.4788 & .050 & 5.4576 & .050 & 5.5554 & .049 & 5.5378 & .050 \\
\hline 0.020 & 7.6875 & .020 & 7.4701 & .021 & 7.6777 & .0200 & 7.5263 & .021 & 7.6948 & .019 & 7.5875 & .020 \\
\hline 0.010 & 9.5134 & .010 & 9.0867 & .011 & 9.4325 & .010 & 9.0514 & .010 & 9.2269 & .010 & 9.0567 & .011 \\
\hline \multirow[t]{2}{*}{0.005} & 11.1861 & .0049 & 10.1608 & .0059 & 11.5446 & .0043 & 10.5105 & .0052 & 10.845 & .0046 & 10.5965 & .0054 \\
\hline & & $\mathrm{m}=3$ & $\mathrm{n}=24$ & & & $\mathrm{~m}=3$ & $\mathrm{n}=25$ & & & $\mathrm{~m}=3$ & $\mathrm{n}=26$ & \\
\hline 0.200 & 2.9346 & .198 & 2.9305 & .200 & 2.9230 & 199 & 2.9196 & .200 & 2.9518 & 200 & 2.9288 & .200 \\
\hline 0.100 & 3.9901 & .099 & 3.9696 & .100 & 3.9645 & .100 & 3.9645 & .101 & 3.9828 & .100 & 3.9775 & .100 \\
\hline 0.050 & 5.5686 & .050 & 5.5463 & .051 & 5.5424 & .049 & 5.4941 & .050 & 5.6001 & .050 & 5.5736 & .050 \\
\hline 0.020 & 7.6349 & .019 & 7.5029 & .021 & 7.6441 & .020 & 7.6152 & .020 & 7.7392 & .020 & 7.6155 & .020 \\
\hline 0.010 & 9.4075 & .010 & 9.1548 & .010 & 9.1838 & .010 & 9.0769 & .010 & 9.1764 & .010 & 9.0829 & .010 \\
\hline \multirow[t]{2}{*}{0.005} & 10.8813 & .0048 & 10.6064 & .0055 & 10.9392 & .0049 & 10.4910 & .0055 & 10.6898 & .0049 & 10.6528 & .0055 \\
\hline & & $\mathrm{m}=4$ & $n=4$ & & & $\mathrm{~m}=4$ & $\mathrm{n}=5$ & & & $\mathrm{~m}=4$ & $n=6$ & \\
\hline 0.200 & 4.0833 & .143 & 3.9833 & .257 & 3.4088 & .198 & 3.4088 & .214 & 3.5681 & .190 & 3.4375 & .200 \\
\hline 0.100 & 5.3333 & .057 & 4.3333 & .114 & 4.0353 & .087 & 3.9885 & .103 & 4.5284 & .095 & 4.1363 & .105 \\
\hline 0.050 & & & 5.3333 & .057 & 5.4285 & .040 & 5.2322 & .056 & 4.8465 & .048 & 4.8181 & .057 \\
\hline 0.020 & & & 5.3333 & .057 & 6.3636 & .008 & 6.1298 & .024 & 7.0000 & .014 & 6.4375 & .024 \\
\hline 0.010 & & & 5.3333 & .057 & 6.3636 & .008 & 6.1298 & .024 & 7.2727 & .005 & 7.0000 & .014 \\
\hline \multirow[t]{2}{*}{0.005} & & & 5.3333 & .057 & & & 6.3636 & .008 & 7.2727 & .0048 & 7.0000 & .014 \\
\hline & & $\mathrm{m}=4$ & $\mathrm{n}=7$ & & & $\mathrm{~m}=4$ & $\mathrm{n}=8$ & & & $\mathrm{~m}=4$ & $\mathrm{n}=9$ & \\
\hline 0.200 & 3.3150 & .197 & 3.2637 & .203 & 3.3681 & .198 & 3.3269 & .202 & 3.2467 & .194 & 3.2467 & .200 \\
\hline 0.100 & 4.3809 & .094 & 4.3260 & .100 & 4.2706 & .099 & 4.2417 & .103 & 4.2337 & .099 & 4.1991 & .105 \\
\hline 0.050 & 5.4065 & .045 & 5.3809 & .052 & 5.1442 & .048 & 5.0824 & .053 & 5.5064 & .046 & 5.3766 & .052 \\
\hline 0.020 & 7.3589 & .015 & 6.5897 & .021 & 6.3804 & .018 & 6.3804 & 0.022 & 6.9610 & .018 & 6.9350 & .021 \\
\hline 0.010 & 7.8974 & .009 & 7.3589 & .015 & 8.7012 & .006 & 8.1978 & .010 & 8.2337 & .010 & 7.1428 & .013 \\
\hline \multirow[t]{2}{*}{0.005} & 8.0769 & .0030 & 7.8974 & .0091 & & & 8.7912 & .0061 & 9.4285 & .0042 & 8.9610 & 0070 \\
\hline & & $\mathrm{m}=4$ & $\mathrm{n}=10$ & & & $\mathrm{~m}=4$ & $\mathrm{n}=11$ & & & $m=4$ & $\mathrm{n}=12$ & \\
\hline 0.200 & 3.2362 & .199 & 3.2050 & .201 & 3.2417 & .200 & 3.2056 & .201 & 3.2324 & .200 & 3.1799 & .201 \\
\hline 0.100 & 4.2450 & .099 & 4.2262 & .101 & 4.2284 & .099 & 4.1673 & .100 & 4.2535 & .100 & 4.2156 & .101 \\
\hline 0.050 & 5.3450 & .049 & 5.3362 & .051 & 5.2468 & .050 & 5.2347 & .051 & 5.3403 & .050 & 5.2913 & .052 \\
\hline 0.020 & 7.0050 & .019 & 6.5362 & .021 & 6.8384 & .020 & 6.7829 & .021 & 7.0168 & .019 & 7.0035 & .020 \\
\hline 0.010 & 7.8862 & .009 & 7.6762 & .013 & 8.2702 & .010 & 8.2625 & .011 & 8.7682 & .009 & 8.3375 & .010 \\
\hline \multirow[t]{2}{*}{0.005} & 9.6562 & .0050 & 9.0262 & .0070 & 10.2907 & .0037 & 9.7909 & .0051 & 9.3060 & .0049 & 8.9698 & .0060 \\
\hline & & $\mathrm{m}=4$ & $n=13$ & & & $\mathrm{~m}=4$ & $\mathrm{n}=14$ & & & $\mathrm{~m}=4$ & $\mathrm{n}=15$ & \\
\hline 0.200 & 3.1821 & .200 & 3.1713 & .200 & 3.1729 & .200 & 3.1701 & .200 & 3.1781 & .200 & 3.1734 & .201 \\
\hline 0.100 & 4.1970 & .100 & 4.1781 & .102 & 4.1513 & .100 & 4.1393 & .100 & 4.1361 & .099 & 4.1280 & .100 \\
\hline 0.050 & 5.3630 & .049 & 5.3576 & .050 & 5.2953 & .050 & 5.2615 & .051 & 5.3610 & .050 & 5.3381 & .050 \\
\hline 0.020 & 7.1794 & .019 & 7.0958 & .020 & 7.2302 & .020 & 7.1506 & .020 & 7.3428 & .020 & 7.3045 & .020 \\
\hline 0.010 & 8.2294 & .010 & 8.1727 & .011 & 8.4144 & .009 & 8.3009 & .010 & 8.5902 & .010 & 8.5122 & .010 \\
\hline 0.005 & 9.6545 & .0046 & 9.5330 & .0055 & 9.9476 & .0049 & 9.6938 & .0056 & 10.1045 & .0049 & 9.7811 & .0054 \\
\hline
\end{tabular}


Table 2 (cont.)

\begin{tabular}{|c|c|c|c|c|c|c|c|c|c|c|c|c|}
\hline$\alpha$ & $\underline{w}$ & $\underline{p}$ & $\bar{w}$ & $\bar{p}$ & $\underline{w}$ & $\underline{p}$ & $\bar{w}$ & $\bar{p}$ & $\underline{w}$ & $\underline{p}$ & $\bar{w}$ & $\bar{p}$ \\
\hline & & $m=4$ & $\mathrm{n}=16$ & & & $m=4$ & $\mathrm{n}=17$ & & & $\mathrm{~m}=4$ & $\mathrm{n}=18$ & \\
\hline 0.200 & 3.1769 & 0.199 & 3.1669 & .200 & 3.1482 & .200 & 3.1464 & .201 & 3.1530 & .200 & 3.1413 & .200 \\
\hline 0.100 & 4.1680 & .100 & 4.1661 & .101 & 4.2149 & .100 & 4.2096 & .100 & 4.1820 & .099 & 4.1775 & .100 \\
\hline 0.050 & 5.3877 & .050 & 5.3779 & .051 & 5.4040 & .050 & 5.4038 & .050 & 5.3659 & .050 & 5.3559 & .050 \\
\hline 0.020 & 7.3336 & .020 & 7.2881 & .020 & 7.3345 & .020 & 7.2867 & .020 & 7.2789 & .0202 & 7.2681 & .020 \\
\hline 0.010 & 8.5665 & .010 & 8.5638 & .010 & 8.6124 & .010 & 8.5756 & .010 & 8.7934 & .010 & 8.7173 & .010 \\
\hline \multirow[t]{2}{*}{0.005} & 10.0103 & .0047 & 9.8625 & .0052 & 9.9360 & .0048 & 9.7931 & .0052 & 10.1821 & .0048 & 10.1168 & .0051 \\
\hline & & $m=4$ & $\mathrm{n}=19$ & & & $m=4$ & $\mathrm{n}=20$ & & & $m=4$ & $\mathrm{n}=21$ & \\
\hline 0.200 & 3.1586 & .200 & 3.1398 & .200 & 3.1397 & .200 & 3.1373 & .200 & 3.1371 & .200 & 3.1313 & .200 \\
\hline 0.100 & 4.1541 & 100 & 4.1436 & .100 & 4.1493 & 100 & 4.1479 & 100 & 4.1464 & .100 & 4.1330 & .100 \\
\hline 0.050 & 5.3977 & .049 & 5.3751 & .050 & 5.3985 & .050 & 5.3844 & .050 & 5.4134 & .050 & 5.4077 & .050 \\
\hline 0.020 & 7.3112 & .020 & 7.2699 & .020 & 7.3358 & .020 & 7.3185 & .020 & 7.4488 & .020 & 7.4122 & .020 \\
\hline 0.010 & 8.7481 & .010 & 8.7225 & .010 & 8.7746 & .010 & 8.7486 & .010 & 8.9051 & .010 & 8.8223 & .010 \\
\hline \multirow[t]{2}{*}{0.005} & 10.2226 & .0049 & 10.1015 & .0051 & 10.2820 & .0050 & 10.1472 & .0052 & 10.2684 & .0049 & 10.2359 & .0051 \\
\hline & & $m=5$ & $\mathrm{n}=5$ & & & $m=5$ & $\mathrm{n}=6$ & & & $\mathrm{~m}=5$ & $\mathrm{n}=7$ & \\
\hline 0.200 & 3.5290 & .198 & 3.2618 & .214 & 3.4017 & .197 & $\mathbf{3 . 3 3 3 3}$ & .201 & 3.3827 & .199 & 3.3450 & .202 \\
\hline 0.100 & 4.4618 & .095 & 4.1836 & .111 & 4.5094 & .097 & 4.4273 & .102 & 4.5054 & .098 & 4.4376 & .101 \\
\hline 0.050 & 5.1818 & .048 & 4.8381 & .063 & 5.0735 & .050 & 5.0427 & .058 & 5.4474 & .048 & 5.3871 & .051 \\
\hline 0.020 & 6.8181 & .008 & 6.4090 & .024 & 6.2940 & .019 & 6.0683 & .024 & 6.5852 & .018 & 6.4270 & .020 \\
\hline 0.010 & 6.8181 & .008 & 6.4090 & .024 & 7.4837 & .009 & 6.8376 & .011 & 7.4442 & .008 & 7.3538 & .010 \\
\hline \multirow[t]{2}{*}{0.005} & & & 6.8181 & .008 & 7.6068 & .0043 & 7.4837 & .0087 & & & 8.4615 & .0051 \\
\hline & & $m=5$ & $\mathrm{n}=8$ & & & $\mathrm{~m}=5$ & $\mathrm{n}=9$ & & & $m=5$ & $\mathrm{n}=10$ & \\
\hline 0.200 & 3.2961 & .197 & 3.2961 & .202 & 3.2900 & .200 & 3.2622 & .201 & 3.3292 & .200 & 3.3143 & .200 \\
\hline 0.100 & 4.4026 & .096 & 4.3246 & .106 & 4.4666 & .095 & 4.4100 & .102 & 4.3595 & .100 & 4.3405 & .101 \\
\hline 0.050 & 5.4935 & .046 & 5.3532 & .051 & 5.4444 & .050 & 5.4233 & .051 & 5.3404 & .050 & 5.3241 & .050 \\
\hline 0.020 & 6.7558 & .018 & 6.4285 & .021 & 6.6944 & .020 & 6.6400 & .021 & 6.8762 & .020 & 6.7239 & .020 \\
\hline 0.010 & 7.4415 & .009 & 7.3168 & .011 & 7.7777 & .010 & 7.5600 & .011 & 7.6948 & .010 & 7.6066 & .011 \\
\hline \multirow[t]{2}{*}{0.005} & 8.3532 & .0047 & 8.2987 & .0062 & 8.2711 & .0050 & 8.2400 & .0060 & 8.9092 & .0047 & 8.8310 & .0053 \\
\hline & & $\mathrm{m}=5$ & $\mathrm{n}=11$ & & & $\mathrm{~m}=5$ & $\mathrm{n}=12$ & & & $\mathrm{~m}=5$ & $\mathrm{n}=13$ & \\
\hline 0.200 & 3.2895 & .200 & 3.2565 & .200 & 3.2467 & .200 & 3.2444 & .200 & 3.2576 & .200 & 3.2576 & .200 \\
\hline 0.100 & 4.3676 & .100 & 4.3590 & .100 & 4.3321 & .100 & 4.3251 & .101 & 4.3295 & .100 & 4.3216 & .100 \\
\hline 0.050 & 5.4004 & .050 & 5.3943 & .051 & 5.4362 & .050 & 5.4339 & .050 & 5.4002 & .050 & 5.4002 & .050 \\
\hline 0.020 & 6.7731 & .020 & 6.7034 & .020 & 6.9356 & .020 & 6.8584 & .020 & 6.9724 & .020 & 6.9305 & .020 \\
\hline 0.010 & 8.0235 & .010 & 7.9123 & .010 & 8.2432 & .010 & 8.1988 & .010 & 8.2860 & .010 & 8.1773 & .010 \\
\hline \multirow[t]{2}{*}{0.005} & 9.4096 & .0046 & 9.1162 & .0050 & 9.1812 & .0048 & 8.9286 & .0052 & 9.3724 & .0049 & 9.2534 & .0051 \\
\hline & & $\mathrm{m}=5$ & $\mathrm{n}=14$ & & & $\mathrm{~m}=5$ & $\mathrm{n}=15$ & & & $\mathrm{~m}=6$ & $\mathrm{n}=6$ & \\
\hline 0.200 & 3.2444 & .200 & 3.2438 & .200 & 3.2299 & .200 & 3.2294 & .200 & 3.4981 & .199 & 3.2674 & .203 \\
\hline 0.100 & 4.3277 & .100 & 4.3228 & .100 & 4.3187 & .100 & 4.3173 & .100 & 4.4981 & .100 & 4.4835 & .104 \\
\hline 0.050 & 5.4038 & .050 & 5.3989 & .050 & 5.4186 & .050 & 5.4154 & .050 & 5.4212 & .048 & 5.4212 & .052 \\
\hline 0.020 & 7.0441 & .020 & 7.0403 & .020 & 7.0269 & .020 & 7.0255 & .020 & 6.3553 & .019 & 6.1648 & .024 \\
\hline 0.010 & 8.2403 & .010 & 8.1971 & .010 & 8.4011 & .010 & 8.3831 & .010 & 7.4102 & .006 & 7.2527 & .011 \\
\hline 0.005 & 9.5495 & .0050 & 9.4789 & .0052 & 9.5897 & .0049 & 9.5786 & .0050 & 8.0109 & .0043 & 7.4102 & .0065 \\
\hline
\end{tabular}


Table 2 (cont.)

\begin{tabular}{|c|c|c|c|c|c|c|c|c|c|c|c|c|}
\hline$\alpha$ & $\underline{w}$ & $\underline{p}$ & $\bar{w}$ & $\bar{p}$ & $\underline{w}$ & $\underline{p}$ & $\bar{w}$ & $\bar{p}$ & $\underline{w}$ & $\underline{p}$ & $\bar{w}$ & $\bar{p}$ \\
\hline & & $\mathrm{m}=6$ & $\mathrm{n}=7$ & & & $m=6$ & $\mathrm{n}=8$ & & & $m=6$ & $=9$ & \\
\hline 0.200 & 3.3543 & .200 & 3.3321 & .203 & 3.3500 & .197 & 3.3375 & .201 & 3.2953 & .199 & 3.2843 & .200 \\
\hline 0.100 & 4.4749 & .100 & 4.4452 & .101 & 4.4468 & .100 & 4.4385 & .101 & 4.5037 & .100 & 4.5020 & .100 \\
\hline 0.050 & 5.4545 & .048 & 5.4545 & .050 & 5.4333 & .050 & 5.4218 & .051 & 5.4449 & .050 & 5.4296 & .0050 \\
\hline 0.020 & 6.5677 & .019 & 6.3747 & .020 & 6.5302 & .020 & 6.5218 & .020 & 6.7825 & .020 & 6.7569 & .020 \\
\hline 0.010 & 7.2727 & .010 & 6.9758 & .011 & 7.3375 & .010 & 7.2708 & .011 & 7.5588 & .010 & 7.4612 & .010 \\
\hline \multirow[t]{2}{*}{0.005} & 8.2300 & .0041 & 8.0222 & .0051 & 8.3218 & .0047 & 8.1968 & .0053 & 8.2953 & .0050 & 8.2782 & .0054 \\
\hline & & $\mathrm{m}=6$ & $\mathrm{n}=10$ & & & $\mathrm{~m}=6$ & $\mathrm{n}=11$ & & $\mathrm{~m}=6 \quad \mathrm{n}=12$ & & & \\
\hline$\overline{0.200}$ & 3.3170 & .200 & 3.3086 & .201 & 3.2812 & .200 & 3.2748 & .202 & 3.2998 & .200 & 3.2998 & .200 \\
\hline 0.100 & 4.4683 & .100 & 4.4487 & .101 & 4.4572 & .100 & 4.4550 & .100 & 4.4561 & .100 & 4.4473 & .100 \\
\hline 0.050 & 5.4700 & .049 & 5.4683 & .050 & 5.4694 & .050 & 5.4630 & .051 & 5.5087 & .050 & 5.5065 & .050 \\
\hline 0.020 & 6.8313 & .020 & 6.8016 & .020 & 6.8474 & .020 & 6.8410 & .020 & 6.8837 & .020 & 6.8771 & .020 \\
\hline 0.010 & 7.7563 & .010 & 7.6605 & .010 & 7.8872 & .010 & 7.8745 & .010 & 7.9539 & .010 & 7.9100 & .010 \\
\hline \multirow[t]{2}{*}{0.005} & 8.5019 & .0049 & 8.4733 & .0054 & 8.9718 & .0048 & 8.8718 & .0050 & 9.0235 & .0050 & 8.9183 & .0051 \\
\hline & & $\mathrm{m}=7$ & $\mathrm{n}=7$ & & & $\mathrm{~m}=7$ & $\mathrm{n}=8$ & & & $\mathrm{~m}=7$ & $=9$ & \\
\hline 0.200 & 3.3020 & .200 & 3.2857 & .203 & 3.3223 & .200 & 3.3189 & .200 & 3.3445 & .198 & 3.3328 & .200 \\
\hline 0.100 & 4.4653 & .100 & 4.4500 & .101 & 4.4931 & .100 & 4.4921 & .100 & 4.5012 & .100 & 4.4756 & .100 \\
\hline 0.050 & 5.5602 & .049 & 5.5551 & .050 & 5.5029 & .050 & 5.5029 & .050 & 5.5694 & .050 & 5.5480 & .050 \\
\hline 0.020 & 6.7183 & .020 & 6.6163 & .021 & 6.7162 & .020 & 6.6980 & .020 & 6.7635 & .020 & 6.7581 & .020 \\
\hline 0.010 & 7.2500 & .010 & 7.0499 & .011 & 7.4921 & .010 & 7.4686 & .010 & 7.5478 & .010 & 7.5424 & .010 \\
\hline \multirow[t]{2}{*}{0.005} & 8.0500 & .0047 & 7.8622 & .0058 & 8.0855 & .0048 & 7.9873 & .0051 & 8.4740 & .0047 & 8.4441 & .0051 \\
\hline & & $\mathrm{m}=7$ & $\mathrm{n}=10$ & & & $\mathrm{~m}=7$ & $\mathrm{n}=11$ & & & $\mathrm{~m}=7$ & $=12$ & \\
\hline 0.200 & 3.3223 & .199 & 3.2982 & .201 & 3.2836 & .200 & 3.2831 & .200 & 3.2905 & .200 & 3.2871 & .201 \\
\hline 0.100 & 4.5132 & .100 & 4.4972 & .100 & 4.5140 & .100 & 4.5140 & .100 & 4.4804 & .100 & 4.4789 & .101 \\
\hline 0.050 & 5.5739 & .050 & 5.5719 & .050 & 5.5428 & .050 & 5.5428 & .050 & 5.6012 & .050 & 5.6000 & .050 \\
\hline 0.020 & 6.8912 & .020 & 6.8711 & .020 & 6.8373 & .020 & 6.8373 & .020 & 6.9289 & .020 & 6.9159 & .020 \\
\hline 0.010 & 7.7453 & .010 & 7.7052 & .010 & 7.7821 & .010 & 7.7805 & .010 & 7.9493 & .010 & 7.9373 & .010 \\
\hline \multirow[t]{2}{*}{0.005} & 8.5894 & .0050 & 8.5714 & .0051 & 8.7792 & .0050 & 8.7648 & .0050 & 8.9306 & .0050 & 8.9087 & .0050 \\
\hline & & $\mathrm{m}=8$ & $\mathrm{n}=8$ & & & $\mathrm{~m}=8$ & $\mathrm{n}=9$ & & & $\mathrm{~m}=8$ & $=10$ & \\
\hline 0.200 & 3.3161 & .199 & 3.3114 & .200 & 3.3294 & .199 & 3.3138 & .200 & 3.2947 & .200 & 3.2922 & .200 \\
\hline 0.100 & 4.5215 & .099 & 4.5063 & .101 & 4.5126 & .100 & 4.4990 & .100 & 4.5401 & .100 & 4.5401 & .100 \\
\hline 0.050 & 5.5567 & .050 & 5.5509 & .050 & 5.6062 & .050 & 5.6042 & .050 & 5.6291 & .050 & 5.6236 & .050 \\
\hline 0.020 & 6.8413 & .020 & 6.7904 & .020 & 6.8323 & .020 & 6.8245 & .020 & 6.9243 & .020 & 6.9159 & .020 \\
\hline 0.010 & 7.6685 & .010 & 7.5215 & .010 & 7.6510 & .010 & 7.6413 & .010 & 7.7567 & .010 & 7.7552 & .010 \\
\hline \multirow[t]{2}{*}{0.005} & 8.2352 & .0050 & 8.1060 & .0053 & 8.4580 & .0050 & 8.4541 & .0051 & 8.6375 & .0050 & 8.6138 & .0050 \\
\hline & & $\mathrm{m}=9$ & $\mathrm{n}=9$ & & & $\mathrm{~m}=9$ & $\mathrm{n}=10$ & & & $\mathrm{~m}=10$ & $=10$ & \\
\hline 0.200 & 3.3001 & .200 & 3.2938 & .200 & 3.2831 & .200 & 3.2815 & .200 & 3.2938 & .200 & 3.2922 & .200 \\
\hline 0.100 & 4.5414 & .100 & 4.5321 & .100 & 4.5346 & .100 & 4.5308 & .100 & 4.5354 & .100 & 4.5224 & .100 \\
\hline 0.050 & 5.6218 & .050 & 5.6174 & .050 & 5.6481 & .050 & 5.6468 & .100 & 5.6922 & .050 & 5.6862 & .050 \\
\hline 0.020 & 6.8923 & .020 & 6.8849 & .020 & 6.9801 & .020 & 6.9779 & .020 & 7.0358 & .020 & 7.0311 & .020 \\
\hline 0.010 & 7.7602 & .010 & 7.7500 & .010 & 7.8680 & .010 & 7.8563 & .010 & 7.9567 & .010 & 7.9475 & .010 \\
\hline 0.005 & 8.5224 & .0050 & 8.4931 & .0051 & 8.7150 & .0050 & 8.7101 & .0050 & 8.7702 & .0050 & 8.7657 & .0050 \\
\hline
\end{tabular}

